\title{
Homeopathy for Pain Management
}

\section{Burke Lennihan, RN, CCH}

\section{Abstract}

Homeopathy is often overlooked as a modality for pain management. However, it deserves to be a first-line treatment due to its safety, effectiveness, and cost-effectiveness. Limitations to the acceptance of homeopathy in the United States include physicians' lack of familiarity with the research studies, which primarily come from Europe, where homeopathy is integrated into the national healthcare systems of nearly all European countries. The database of the social security system in France, where citizens can choose a homeopathic or conventional family doctor, shows that the former modality provides comparable results in pain management while significantly reducing the use of conventional painkillers. Research in the United States is minimal due to lack of government or industry funding. In addition, exaggerated media reports as to the potential harm from homeopathy have resulted in undue reluctance to use it among both physicians and the general public. A large-scale review of the German drug safety database has revealed a miniscule number of adverse events. Finally, resistance to the use of homeopathy - based on the mistaken notion that it contains nothing but water-is addressed by citations from the newly emerging field of ultrahigh dilution physics. A description of the most common homeopathic medicines for various types of pain is provided, including back pain, dental pain, labor pains, and trigeminal neuralgia. Homeopathic medicines must be customized to the individual patient in chronic conditions. Yet, a severe acute trauma can respond to the same homeopathic medicine in nearly all individuals. As an example, a standard protocol is provided to speed healing and reduce pain after surgery or dental extraction. While homeopathic medicines are rarely tested against conventional painkillers in a head-to-head trial, certain research studies show that homeopathic medicines can enhance healing for those already on conventional medications and can reduce pain in a condition such as a fracture for which effective drug treatment is lacking.

Keywords: homeopathy, pain management, acute pain, back pain, musculoskeletal pain, postoperative pain

(C) Burke Lennihan 2017; Published by Mary Ann Liebert, Inc. This Open Access article is distributed under the terms of the Creative Commons Attribution Noncommercial License (http://creativecommons .org/licenses/by-nc/4.0/) which permits any noncommercial use, distribution, and reproduction in any medium, provided the original author(s) and the source are cited.

\section{Introduction}

Pain management has been called "the leading edge" of complementary and alternative medicine (CAM) modalities for integration into conventional healthcare in the United States. ${ }^{1}$ New pain-management guidelines from the American College of Physicians and the American Pain Society recommend a wide range of CAM modalities instead of opioids for chronic low back pain - but the recommended CAM modalities do not include homeopathy. ${ }^{2}$ In contrast, the American Pain Management Association's textbook, Weiner's Pain Management, has a detailed chapter on homeopathic treatment of pain syndromes. ${ }^{3}$

In a large-scale study in France, where national health insurance covers homeopathic as well as conventional primarycare doctors, the former prescribed fewer opioids (and half as many nonsteroidal anti-inflammatory drugs [NSAIDs]) for musculoskeletal disorders while providing better results in terms of pain reduction and quality of life. ${ }^{4}$ Further, there is some evidence to suggest that homeopathy can work faster than conventional pain medications or reduce the need for them, ${ }^{5,6}$ without danger of dependence or withdrawal symptoms. ${ }^{7}$ There is also research to suggest that homeopathy can work better than placebo in reducing pain, for example from endometriosis, ${ }^{8}$ joint pain and stiffness, ${ }^{9}$ and hemorrhoids. ${ }^{10}$

For those in the throes of withdrawal, homeopathic medicines can minimize suffering and help to ensure a successful rehabilitation outcome, as reported in the forthcoming article on PostAcute-Withdrawal Syndrome. (Once dependency is established, it can be too late to substitute homeopathy for opioids because the receptors have been altered; L. Butehorn, pers. commun., 2017).

\section{Advantages of Homeopathy}

Homeopathy is part of mainstream medicine in most countries in Europe and Latin America as well as in India and Russia. Most of its medicines are classified as over-the-counter (OTC) drugs by the Food and Drug Administration (FDA). Their safety record has not required restriction to prescription-only status.*

\footnotetext{
*A few homeopathic medicines have prescription-only status because of their source material (Opium, Cannabis) or because they have no indications for self-care (Crataegus, used homeopathically only for heart conditions; Crataegus or hawthorn as a herbal extract is available OTC in health-food stores as a traditional heart tonic). ${ }^{7}$
} 
In one large study, Germany's drug safety database was searched for adverse reports on $>300$ million doses of homeopathic and anthroposophical drugs (similar to homeopathy). There were only 486 reports of adverse events, of which only 46 were verified as serious. ${ }^{11}$ The accompanying article on the regulation of homeopathy contains a detailed examination of the FDA's recall of Zicam ${ }^{\circledR}$ and teething tablets, the only two homeopathic products of recent safety concern. These seem to be examples of disproportionate negative media rather than of serious safety concerns with homeopathy.

Homeopathy's mechanism of action is currently being revealed by studies in the newly emerging field of ultrahigh dilution physics. In one scenario, nanoparticles of the original medicinal substance ${ }^{12}$ attract water molecules to form liquid crystal structures in the dilution water, which in turn convey information that triggers a homeostatic reaction in the body. ${ }^{13}$ In another, coherent domains are formed "among the molecules of the water-alcohol solvent, as predicted by quantum electrodynamics." ${ }^{~ 14,15}$ The nanodose levels that persist in homeopathic medicines are comparable to the levels at which the body's hormones and cell-signaling agents operate. These two qualities of homeopathy-the active ingredient being present only in nanoparticles and the mechanism of action based on conveying information rather than its medicinal substance-give homeopathic medicines their unique qualities: the patient needs less and less of them over time; they do not create dependency or withdrawal; they do not interact with other medications; and they can heal other health conditions at the same time in addition to the chief complaint, that is, they provide side benefits instead of side effects. ${ }^{7}$

Homeopathic medicines in their pure form are not patentable, which leads to another benefit: they are extremely inexpensive, costing in the range of pennies per treatment. A clinic can be equipped with a year's supply for $<\$ 1,000$. Because they are non-addictive and have no resale value, there is no incentive for robbery, which enhances the safety of a clinic or pharmacy. They can safely be administered by a community health worker or health coach - which can also be done legally because of their OTC status. A clinic can thus save on salary expenses as well as drug costs.

As an example of this concept in action, Homeopaths Without Borders is empowering Haitians as "homéopathes communautaires" to conduct their own local homeopathy clinics with the approval and supervision of the Haitian Health Ministry. ${ }^{16}$

\section{Practical Protocols for Post-Surgery/Post-Dental Extraction Pain Management}

The use of Arnica before and after surgery to reduce bruising, swelling, soreness, and pain ${ }^{6,17}$ has been documented, with some variation in the potency (strength) and frequency of dosage.
Arnica can be used in the moderate 30c potency found in retail stores. ${ }^{\dagger}$ A typical dose for all of the medicines described in this article is two pellets dissolved in the mouth. In addition to Arnica, the following medicines are also used:

- Calendula to help approximate the wound, ${ }^{18}$ prevent scarring and infection, and reduce pain. (One study on breast cancer patients showed that it reduced pain from radiation-induced dermatitis, as well as helping it to heal. $)^{19}$

- Hypericum to promote wound healing ${ }^{18}$ and reduce nerve pain.

- Phosphorus to help stop excessive bleeding and to help the person come out of anesthesia.

This protocol takes advantage of homeopathy's ability to work preventively when given right before it is needed.

- The morning of surgery (or the night before if surgery is in the early morning), one dose of Arnica 30c. Because the pellets dissolve quickly in the mouth and are not swallowed, they do not violate nip per os restrictions.

- Soon after surgery, a dose of Phosphorus 30c, only if the person is drowsy, disoriented, or nauseous after anesthesia, or if there is excessive bleeding (e.g., after a tooth extraction).

- For the first three days, Arnica 30c three times a day. Then Calendula 30c three times a day until the wound is healed.

- If there is nerve pain (the patient reports pain shooting along the path of a nerve, or pain like an electric shock, or pins and needles, or numbness), give Hypericum 30c three times a day until resolved.

- If there is nerve damage, injury to the spine, or a headache following an epidural or spinal tap, give Hypericum as well. ${ }^{20}$

If symptoms are very severe, the medicine can be given more frequently, as often as hourly. If a family member wants to bring the medicines to their loved one in the hospital, an easy way to do this is to dissolve two pellets in a small bottle of water (about 4 ounces, $125 \mathrm{~mL}$ ) and have the patient sip on it occasionally, labeling what each bottle of water is for. If the person is unconscious, the water can be dabbed on their lips. The water solution of Calendula can be used to irrigate the wound and dampen the gauze in a wet-to-dry dressing in order to speed healing.

\footnotetext{
${ }^{\dagger}$ Potency in homeopathy is based on the number of times the starting substance has been put through the serial dilution/activation process, with higher numbers representing stronger action because of the increased activation. A potency of $30 \mathrm{c}$ is not to be confused with $30 \mathrm{cc}$, a measure of fluid volume used for conventional drugs. European countries use the convention $\mathrm{CH}$ rather than $\mathrm{c}$ to indicate potency. Some of the citations in this study refer to $30 \mathrm{CH}$, for example, which is comparable to the 30c potency in the United States.
} 
Of course, this is the ideal scenario, and it may not be practical in a hospital unless a physician writes an order for the medicines. Sometimes family members are able to bring the medicines in; in other cases, they have to wait until the patient comes home. The sooner the protocol can be started, the more effective it will be.

Homeopathic Calendula can be difficult to find in stores. With advance notice, it can be ordered online. In an emergency situation, herbal Calendula can be used instead, diluted in a 1:20 ratio. A homeopathic preparation of a nontoxic herb such as Calendula can enhance its action and also allow for healing from the inside out. For example, if someone has extensive first-degree burns or large areas of abrasion, dissolving homeopathic Calendula in the mouth can be as effective as applying it topically over a large area. The oral administration can be simpler and less painful. For a tooth extraction, the same protocol can be followed, with the possible addition of the medicines for dry socket described under Toothache below.

In my own experience and that of my colleagues, our clients have survived surgery and tooth extraction with rapid healing and minimal need for conventional pain medications. Patients frequently report not needing any patient-controlled analgesia (PCA) after surgery.

One of my colleagues, a pharmacist, provided this protocol for her elderly mother who was having a bowel resection. Her mother's care team was surprised to find her trying to climb over the bed rails and head toward the bathroom the day after major abdominal surgery. I have also had clients who had a wisdom tooth removed who did not need to fill their prescription for a narcotic painkiller, and clients who needed no PCA after major surgery such as a double mastectomy.

\section{Homeopathic Medicines for Specific Pain Conditions}

A well-known principle of homeopathy is that the medicine must be individualized to the patient: 10 patients with fibromyalgia or rheumatoid arthritis are likely to receive 10 different medicines from a professional homeopath because each patient has a unique pattern of symptoms. However, this primarily applies to chronic conditions.

In an emergency situation, or in the case of a very virulent infectious disease, the nature of the trauma or infection imposes the same symptom pattern on nearly everyone. For example, Arnica is useful in almost all acute cases of softtissue trauma with bruising, swelling, and/or soreness. This makes it possible to create a standardized protocol using Arnica for post-surgery recuperation or for sports injuries. Hypericum, with its symptom set of numbness, tingling, other paresthesias, and electric-shock sensations along a nerve path-especially after a blow to the spine or a nerverich area of the body-similarly becomes a universal remedy when an accident or surgery causes acute nerve pain or nerve damage.
When it comes to short-term or palliative pain management (e.g., treating a sprained ankle or providing temporary relief for arthritic pain), the prescriber typically chooses from among the homeopathic medicines well known historically for clinical effectiveness (perhaps 3-20 top medicines for each condition). ${ }^{21-24}$ Among them, the choice is then based on individual characteristics: does the patient find relief from a heating pad or from an ice pack, for example; do they prefer to limber up an aching joint, or do they need to keep it still? This article will provide a simplified version for each of several types of pain, differentiating among just a few of the best-known medicines in order to make homeopathy accessible for healthcare professionals in other disciplines. This will be followed by a review of the research and finally by resources for further study.

\section{Back Pain and Other Musculoskeletal Disorders}

Studies in Europe show that patients choosing homeopathic care have a much lower use of conventional pain medications than those under conventional care (see the Research section for examples). A variety of homeopathic medicines have been used in the studies. Among those with the greatest clinical evidence of effectiveness are:

- Rhus tox and Ruta grav for joint pain with stiffness that is worse in damp weather, needs to be limbered up, and feels better from the application of heat. These two medicines are among the most effective and can be difficult to differentiate. You may observe patients needing Rhus tox swinging a joint to limber it up (the "rusty gate" syndrome) or restlessly pacing in the waiting room. Rhus tox is the medicine most often used for fibromyalgia. Ruta grav has additional qualities of lameness and weakness, for example those whose knees go out from under them when going downstairs.

- Bryonia is for patients with the opposite symptom: they feel worse from stretching and in fact worse from the slightest motion, even the jarring motion of a heavy tread, so they tend to guard or splint their joints. Bryonia is especially good for the serous membranes. Hence, it is good for bursitis and pleuritis.

- Nux vomica is a top remedy for lower back pain in those with typical Nux vomica symptoms: competitive, ambitious, driven, irritable, apt to use coffee, alcohol, spicy or fried foods, sometimes recreational drugs, therefore apt to have a congested liver.

- Protocol: Using the 30c potency (strength) most commonly available in stores, a dose of two pellets dissolved in the mouth can be repeated every one to three hours, depending on the intensity of the pain. In

\footnotetext{
The common abbreviations are used instead of the full homeopathic names in this article, thus Rhus tox for Rhus toxicodendron, Ruta grav for Ruta graveolens, Calc phos for Calcarea phosphorica, and Hepar sulph for Hepar sulphuris calcareum.
} 
general with homeopathic medicines, the medicine is repeated frequently until it starts to take effect, then slowed down or even stopped until symptoms return, so that the body can heal itself.

\section{Broken Bones}

Homeopathy can speed bone healing, as well as reduce the need for analgesics, according to a study done in India. ${ }^{25}$ These three medicines will cover most aspects of bone healing:

- Symphytum is by far the most common medicine for broken bones. Research shows that it can promote callus formation and speed the union of fractures.

- Ruta grav is used when there is a contusion or bone bruise, or when the predominant sensation is soreness rather than sharp pain along the line of the fracture, as in Symphytum.

- Calc phos is used for growing pains in children and for broken bones that take a long time to heal.

- Protocol: Using the 30c potency (strength) most commonly available in stores, a dose of two pellets dissolved in the mouth once a day.

\section{Labor Pains}

The research on homeopathy in childbirth is primarily limited to the study of just one medicine: Caulophyllum. ${ }^{26,27}$ However, a range of medicines to reduce labor pains are included here based on 150 years of experience and the need to reduce the use of epidurals. Epidurals prolong labor and can trigger a "cascade of interventions" leading to a greater need for an oxytocin injection, a higher rate of instrumental births, and a higher risk of Cesarean section due to fetal distress. ${ }^{28}$

Homeopathic medicines can be given in advance to strengthen the uterus and prepare the woman for labor. In one research study, women given a combination of five homeopathic remedies during the ninth month of pregnancy had a $40 \%$ shorter labor and only $25 \%$ as many complications of labor. ${ }^{29}$

The following homeopathic medicines have a long history of successful use for labor pains among homeopathic obstetricians and midwives. Give a dose of $30 \mathrm{c}$ as often as needed to minimize pain and keep the contractions longer and more efficient. The stronger potency 200c will work better for extreme pain. However, it is recommended only for use by homeopathic midwives and others experienced with using homeopathic medicines during labor (not because the higher potency is dangerous, but because it can be more difficult to discern when to repeat it).

- Aconite for violent, intense, sudden labor pains, especially when the woman is afraid she will die.

- Belladonna for extreme labor pains when the woman has the typical Belladonna symptoms (a wild look in the eyes, reddened cheeks, delirium).

- Caulophyllum for when both the labor pains and the woman's moods are quickly changeable: the labor pains seem to flit here and there, the woman is having mood swings.
- Chamomilla for when the pain is so unbearable, nothing can satisfy her: she wants to order everyone out of the room, or she demands something, and as soon as it is given, she refuses it and demands something else.

- Cimicifuga for when the labor pains radiate down the thighs, the woman feels gloomy "as if a black cloud is over her," and she may say she feels she is going insane.

- Kali carbonicum for back labor and needle-like pains.

- Sepia also for back labor with other typical Sepia symptoms such as extreme fatigue leading to weepiness and irritability.

After pains will also respond to some of the same homeopathic medicines:

- Arnica for its typical feeling of bruised soreness and aversion to being touched.

- Chamomilla for when the woman is irritable and dissatisfied, as above.

- Hypericum for pains in the coccyx, and for the effects of an epidural such as a headache.

- Kali carbonicum for needle-like pains in the lower back.

- Pulsatilla for changeable pains and moodiness, including mild weepiness and a desire for consolation and for company in her pain.

\section{Tooth Pain and Other Pain Conditions Treated by Dentists}

- Hypericum 30c, two to four times a day, whenever there is nerve pain, such as following oral surgery or in an impending root-canal situation.

- Chamomilla 30c as often as once an hour for excruciating tooth pain in adults as well as in teething infants.

- Symphytum 30c, once or twice a day, for pain and swelling after dental implant surgery (because Symphytum heals teeth and bones), as well as Arnica, which is always useful for postoperative pain and swelling.

For any toothache and dry socket after a tooth extraction (an extremely painful condition in which the protective blood clot that forms over the empty socket fails to form or is dislodged, exposing nerve endings in the socket):

- Belladonna for throbbing pain.

- Chamomilla for extreme pain relieved by warm drinks and driving the person to angry outbursts at family members and healthcare providers.

- Coffea for agonizing pain relieved by cold water in the mouth, with oversensitivity to any sensory stimulation.

- Hepar sulph if the dry socket becomes infected.

- Silica afterwards to expel the contents of the socket (food debris, pus, etc.).

All of these medicines are available in health-food stores in a $30 \mathrm{c}$ potency and can be given hourly or even more often until 
the excruciating pain is relieved, then three times a day would be a typical dosage when the crisis is over and the patient is on the mend.

The same medicines can be used for an abscessed tooth or indeed any boil or abscess, with the addition of Mercurius, thus:

- Belladonna for the first stage of an abscess, when the area is red, warm, throbbing, and swollen but the pus has not yet gathered.

- Hepar sulph for the second stage when the pus has gathered, and the area is extremely tender and sensitive to exposure to heat or cold, to touch, to the slightest draft, which makes the person touchy and averse to having anyone come close.

- Mercurius is used for the same stage, when the pus has gathered, if several of the typical Mercurius symptoms are present: excess salivation, night sweats, bad breath, and possibly small sores in the mouth, plus the person is extremely sensitive to the temperature in the environment, constantly adjusting heat or air conditioning.

- Silica is used for the last phase, when the pus has come to a head and is ready to drain; Silica will speed the bursting of the abscess and quickly heal the tissues underneath.

I had a client who had no dental insurance and could not afford the root canal that her dentist said was necessary for an abscessed tooth: I gave her these remedies in succession (using Hepar sulph instead of Mercurius because of the exquisite sensitivity of the area). She took them over a period of several days. When she got to the Silica phase, she reported that pus oozed out around the tooth, and the pain disappeared. Her dentist confirmed that she no longer needed the root canal.

\section{Trigeminal Neuralgia}

Trigeminal neuralgia is known as the "suicide disease" because the pain can be so unbearable and so resistant to treatment that it can drive sufferers to suicide. In one small study, homeopathic treatment brought $60 \%$ relief of the pain after four months. ${ }^{30}$

Two of the most common medicines for trigeminal neuralgia can be distinguished simply by which side of the face is in pain: Sanguinaria is used for right-sided trigeminal neuralgia, and Spigelia for left-sided neuralgia. The dosage is 200c (a high potency) every 15 minutes until relief, then wait until relapse before redosing. Remember that these are only two of the possible medicines for the condition. If the first one attempted does not work, a professional homeopath may be able to provide relief with a better-matching medicine.

I had a remarkable experience years ago treating a young woman with long-lasting trigeminal neuralgia so severe that in fact she admitted to contemplating suicide. Her husband was a dentist, so she had had access to the best treatments available in conventional medicine to no avail. Just a few doses of Spigelia 200 c completely relieved her pain. Homeopathy does not usually work so quickly or so well, but when it does, it is memorable.

\section{Headaches}

While research shows that homeopathy can treat headaches successfully, such a wide range of medicines can be useful that it would be impossible to distill them into just a few recommendations. One leading homeopathic reference work lists $>60$ likely medicines for headaches, making a randomized controlled trial difficult if not impossible. ${ }^{31}$ Headaches provide an excellent example of how homeopathic medicines are matched to the individual's symptoms. A homeopath interviewing a patient with frequent headaches will ask what part of the head is affected; whether the left side or right side; what time of day they come on, or what triggers them; whether throbbing or dull; what the pain feels like; and other symptoms specific to the pain. More importantly, the homeopath will ask what stress or trauma might have led to the pattern of repeated headaches. Then the homeopath will choose from among dozens of medicines well known to be effective for headaches.

\section{Research}

This section will review additional research on homeopathy for back pain and dental pain, beginning with a brief look at challenges and limitations to research in homeopathy. In conventional medicine, recent research is given priority. In homeopathy, the same medicines used 200 years ago are still in use today, so that research from the 19th century is still considered valid. ${ }^{32}$

In the United States, very little research is done due to lack of funds (currently little or none; I. Bell, pers. commun., 2017) compared to $\$ 100$ billion a year for conventional medical research. ${ }^{33}$ Homeopathic manufacturers cannot afford to conduct research, since most homeopathic medicines are not patentable. Therefore, the profit is minimal, and the government has stopped funding research in homeopathy. Research on homeopathy is often criticized for small study size and for lack of replication of studies. These issues require substantial funding to be resolved. Due to a lack of funds in the United States, most research is done in Europe, India, and South America, typically funded by governments that cover homeopathy in their national healthcare and therefore are motivated to assess its effectiveness and cost-effectiveness.

Randomized controlled trials (RCTs) are now being called into question in conventional medicine. Professor Sir Michael Rawlins, in his distinguished Harveian Lecture to the Royal College of Physicians of London in 2008, stated:

The [hierarchy of evidence] places RCTs at the highest level with a lesser place for those based on observational studies. Giving such prominence to the results of RCTs, however, is unreasonable. As Bradford Hill, the architect of the RCT, stated so cogently: 'Any belief that the controlled trial is the only way would mean not that the pendulum had swung too far but that it had come right off the hook. ${ }^{, 34} \ldots$ They should be replaced by a diversity of approaches that involve analyzing the totality of the evidence base. ${ }^{35}$ 
Rawlins' lecture provides a detailed analysis of the limitations of RCTs and the value of other forms of research design. Furthermore, as Dr. Marcia Angell, former editor of the New England Journal of Medicine, has observed, RCTs can easily be manipulated in many ways, for example by ending a trial before the time when side effects are known to show up. ${ }^{36}$

RCTs on homeopathy are even more difficult to design correctly, since the nature of the RCT (comparing a single drug to placebo) is not consistent with homeopathy's basic principle of individualizing the medicine to the patient's unique symptoms and circumstances. A single medicine can be used in a protocol such as the post-surgery protocol above, which would make the protocol ideal for an RCT. However, several other variables must be controlled correctly in order for the study to be valid. The medicine must be given in an appropriate potency and at an appropriate frequency, and the endpoints must be chosen appropriately. For example, it would be easy to design a study on Arnica for postsurgical pain in which not enough of the medicine is given, or the study is ended only after the subjects' pain is fully resolved. In both of these cases, the study will appear to prove that Arnica is no more effective than placebo. In chronic conditions, most homeopaths give a strong initial dose. However, this may cause an initial worsening (called an aggravation), which is actually the first sign of healing. A study that lasts only a few weeks may leave the impression that homeopathy worsened the condition.

Another example of research with poor study design is a study on children with upper respiratory infections in Norway, which showed no benefit from homeopathy compared to placebo, in which the choice of medicine was left to parents rather than a professional homeopath - and parents were only allowed to choose from among three out of the two dozen remedies used by professionals. ${ }^{37}$

Similarly, a Spanish study that showed no benefit from homeopathy in otitis media used four homeopathic medicines not commonly used for the condition by professional homeopaths. Two of them are not even included among the 40 medicines listed for the condition in the standard homeopathic reference book, ${ }^{38}$ leaving homeopaths bewildered as to why precious research funds would be spent on this study. Despite the inappropriate choices, fewer children in the homeopathy group experienced a recurrence ( $4.8 \%$ vs. $11.4 \%)$. Yet, the researchers concluded that homeopathy is not an effective adjuvant treatment. ${ }^{39}$

In a British study on Arnica for post-surgical pain, Arnica $6 \mathrm{c}$ did better than placebo on days 2 and 3, as is visible in the graph accompanying the study. ${ }^{40}$ By day 4 , pain was resolved in both groups (everyone had stopped taking their pain medication), and the effect of Arnica 6c was less noticeable. The researchers had chosen pain on day 4 as their endpoint, allowing them to state that Arnica was no better than placebo.

Homeopathy (except for emergencies and acute traumas) does best in a research study on its own terms - allowing a professional homeopath to choose an individualized medicine - and expanding the parameters to include rate of adverse events and of recurrence over an extended period of time, cost of treatment, quality of life, and patient and practitioner satisfaction. The largest outcome study on homeopathy was done by the Swiss government, which concluded that homeopathic treatment was just as effective as conventional treatment while costing $15 \%$ less. ${ }^{41}$ The committee conducted its own research on Swiss citizens' use of homeopathic versus conventional treatment and also examined the existing research, concluding that 20/22 metaanalyses show results for homeopathy greater than placebo.

The following is a review of research on homeopathy for two of the conditions covered in this paper.

\section{Back Pain and Other Musculoskeletal Disorders}

A study in France, where patients can choose whether to go to a conventional or homeopathic doctor, has documented the effectiveness of homeopathy in reducing the use of conventional pain medications. This year-long study compared patients with a variety of musculoskeletal disorders, including osteoarthritis, rheumatism, fibromyalgia, muscle spasms, tendinitis, rotary cuff syndrome, ankylosing spondylitis, intervertebral disc disorders, neck pain, torticollis, and spinal stenosis. The homeopathic patients used $25 \%$ fewer narcotics and only half as many NSAIDs. Not surprisingly, they had fewer adverse events. Yet, there was no difference in specific functional scores and no loss of therapeutic opportunity in the homeopathic cohort-all the more surprising, since the homeopathic cohort's conditions were more longstanding. ${ }^{4}$

A two-year multicenter study in Germany of patients receiving individualized professional homeopathic treatment for chronic low back pain revealed that the severity of their diagnoses was reduced, along with their use of conventional treatments and health services; the number of patients using conventional drugs was half of the baseline, and quality of life improved in both the physical and mental component scale. ${ }^{42}$

Other studies in Europe have shown that homeopathy can provide better relief from back pain than placebo, or comparable pain relief with fewer adverse events. ${ }^{43-45}$

\section{Tooth Pain}

An article in the British Dental Journal ${ }^{46}$ supports the use of homeopathy not only for toothache, but also for other types of pain treated by dentists, including burning mouth syndrome and trigeminal neuralgia. Hypericum in particular (the medicine for nerve pain) was studied in a systematic review that favored its effectiveness, although it was not statistically significant. ${ }^{47}$ Hypericum is more likely to be effective for dental pain involving a nerve rather than pain from soft-tissue trauma from an injury or teething, which could explain its overall apparent lack of effectiveness.

Symphytum 5CH (comparable to the $6 \mathrm{c}$ available in the United States) may reduce pain and swelling after dental implants when given along with conventional pain medication (in an observational study whose authors recommended randomized trials). ${ }^{48}$

Homeopathic treatment was successful in helping $58 \%$ of patients avoid a root canal, tooth extraction, or pulp capping in a small study that used a European method not available in this country (injection of the homeopathic medicine Pulpa Dentis $30 \times) .{ }^{49}$ 


\section{To Contact Burke Lennihan}

Burke Lennihan, RN, $\mathbf{C C H}$

777 Concord Ave., Suite 301

Cambridge, MA 02138

E-mail: blennihan@alumni.harvard.edu

\section{Resources}

\section{General}

Skinner S. An Introduction to Homeopathic Medicine in Primary Care. Gaithersburg, MD: Aspen Publications, 2001. Written by a nurse practitioner, this book will be especially helpful for primary care practitioners.

Ullman D. Evidence-Based Homeopathic Family Medicine. Online document at: www.homeopathic.com. This frequently updated e-book is the best explanation of research on homeopathy, how it works, and its effectiveness for specific conditions, plus a review of the most effective medicines for $>100$ conditions.

\section{For Musculoskeletal Conditions}

Hershoff A. Homeopathy for Musculoskeletal Healing. Berkeley, CA: North Atlantic Books, 1996. Pain relief for headaches, arthritis, and other musculoskeletal conditions, complete with diagrams from a chiropractor/homeopath.

\section{For Labor Pains and Other Maternal-Infant Conditions}

Castro M. Homeopathy for Pregnancy, Birth, and Your Baby's First Year. New York: St. Martins, 1992. Full of wise advice, in addition to an advanced guide to homeopathy in pregnancy.

Moskowitz R. Homeopathic Medicines for Pregnancy and Childbirth. Berkeley, CA: North Atlantic Books, 1992. From a homeopathic physician who began his career working with midwives; useful information and illustrative case studies.

Perko SJ. Homeopathy for the Modern Pregnant Woman and Her Infant. San Antonio: Benchmark, 1997. The most thorough and specific information for labor pains and other maternalinfant health conditions.

\section{For Toothache and Other Dental Symptoms}

Lessell CB. The Dental Prescriber. London: The British Homeopathic Association, 1983.

\section{For Headaches}

Hershoff A. Homeopathy for Musculoskeletal Healing. Berkeley, CA: North Atlantic Books. Provides a differential among the top 15 medicines for headaches with diagrams for parts of the head. However, remember that headaches are especially difficult to treat by this simple approach. There are many more possible medicines for headaches.

Morrison R. Desktop Guide to Physical Pathology. Nevada City, CA: Hahnemann Clinic Publishing, 1998:3-26. Provides a differential among the top 60 remedies for headaches, although it would only be useful for those already trained in homeopathy.

\section{Acknowledgments}

Dana Ullman, $\mathrm{MPH}, \mathrm{CCH}$, provided invaluable assistance in compiling the research studies.

\section{Author Disclosure Statement}

No competing financial interests exist.

\section{References}

1. Weeks J. Academic group leads change for hospitals in integrative pain treatment priorities. Online document at: www.integrativepractitioner.com/ whats-new/news-and-commentary/academic-group-leads-change-hospitalsintegrative-pain-treatment-priorities/ Accessed March 15, 2017.

2. American College of Physicians. Noninvasive treatments for acute, subacute, and chronic low back pain: A clinical practice guideline from the American College of Physicians. Ann Intern Med 2017;166:514-530.

3. Loes M, Ullman D. Homeopathy. In: Boswell MV, Cole BE, eds. Weiner's Pain Management: A Practical Guide for Clinicians, 7th ed. New York: Taylor and Francis, 2006, pp. 1153-1160.

4. Rossignol M, Begaud B, Engel P, et al. Impact of physician preferences for homeopathic or conventional medicines on patients with musculoskeletal disorders: Results from the EP13-MSD cohort. Pharmacoepidemiol Drug Saf 2012;21:1093-1101.

5. Friese KH, Kruse S, Moeller $\mathrm{H}$. The homeopathic treatment of otitis media in children-Comparisons with conventional therapy. Int J Clin Pharmacol Ther 1997;35:296-301.

6. Robertson A, Suryanarayanan R, Banerjee A. Homeopathic Arnica montana for post-tonsillectomy analgesia: A randomised placebo control trial. Homeopathy 2007;96:17-21.

7. American Pharmacists Association. Handbook of Non-Prescription Drugs 15th ed. Washington, DC: American Pharmacists Association, 2006.

8. Teixeira MZ, Podgaec S, Baracat EC. Potentized estrogen in homeopathic treatment of endometriosis pelvic pain: A 24-week, randomized, doubleblind, placebo-controlled study. Eur J Obstet Gynecol Reprod Biol 2017;211: $48-55$.

9. Karp JC, Sanchez C, Guilbert P, et al. Treatment with Ruta graveolens 5CH and Rhus toxicodendron $9 \mathrm{CH}$ may reduce joint pain and stiffness linked to aromatase inhibitors in women with early breast cancer: Results of a pilot observational study. Homeopathy 2016;105:299-308.

10. Das KD, Ghosh S, Das AK, et al. Treatment of hemorrhoids with individualized homeopathy: An open observational pilot study. J Intercult Ethnopharmacol 2016;5:335-342.

11. Jong MC, Jong MU, Bears EW. Adverse drug reactions to anthroposophic and homeopathic solutions for injection: A systematic evaluation of German pharmacovigilance databases. Pharmacoepidemiol Drug Saf 2012;21:1295-1301.

12. Chikramane PS, Suresh AK, Bellare JR, Kane SG. Extreme homeopathic dilutions retain starting materials: A nanoparticulate perspective. Homeopathy 2010;99:231-242

13. Bell I. Pursuing the biological basis of homeopathic remedy response. Online document at: https://youtu.be/U81UZRc8-DU Accessed March 15, 2017. 
14. Sorrentino L, Piraneo S, Riggio E, et al. Is there a role for homeopathy in breast cancer surgery? A first randomized clinical trial on treatment with Arnica montana to reduce post-operative seroma and bleeding in patients undergoing total mastectomy. J Intercult Ethnopharmacol 2017;6:1-8.

15. Marchettini N, Del Giudice E, Voeikov V, Tiezzi E. Water: A medium where dissipative structures are produced by a coherent dynamics. J Theor Biol 2010;265:511-516.

16. Homeopaths Without Borders. Online document at: www.hwbna.org Accessed March 15, 2017.

17. annitti T, Morales-Medina JC, Bellavite P, et al. Effectiveness and safety of Arnica montana in post-surgical setting, pain, and inflammation. Am J Ther 2016;23:e184-197.

18. Hostanska K, Rostock M, Melzer J, Baumgartner S, et al. A homeopathic remedy from Arnica, marigold, St. John's wort and comfrey accelerates in vitro wound scratch closure of NIH $3 \mathrm{~T} 3$ fibroblasts. BMC Complement Altern Med 2012;12:100.

19. Pommier P, Gomez F, Sunyach MP, et al. Phase III randomized trial of Calendula officinalis compared with trolamine for the prevention of acute dermatitis during irradiation for breast cancer. J Clin Oncol 2004;22:1447-1453.

20. Piraneo S, Maier J, Nervetti G, et al. A randomized controlled clinical trial comparing the outcomes of homeopathic-phytotherapeutic and conventional therapy of whiplash in an Emergency Department. Hom Links 2012;25: $50-55$.

21. Hershoff A. Homeopathy for Musculoskeletal Conditions. Berkeley, CA: North Atlantic Books, 1996.

22. Cummings S, Ullman D. Everybody's Guide to Homeopathic Medicines. New York: TarcherPerigree, 2004.

23. Chernin DK. The Complete Homeopathic Resource Guide. Berkeley, CA: North Atlantic Books, 2006.

24. Thomas E. Homeopathy for Sports, Exercise and Dance. Beaconsfield, United Kingdom: Beaconsfield Publishers, 2000.

25. Sharma S, Sharma N, Sharma R. Accelerating the healing of bone fracture using homeopathy: A prospective, randomized double-blind controlled study. BMC Complement Altern Med 2012;12:O61.

26. Eid P, Felisi E, Sideri M. Applicability of homoeopathic caulophyllum thalictroides during labour. Brit Hom J 1993;82:245-248.

27. Eid P, Felisi E, Sideri M. Super-placebo ou action pharmacologique? Une étude en double aveugle, randomisée avec un remedé homéopathique (Caulophyllum thalictroides) dans le travail d'accouchement. Proceedings of the 5th Congress for Homeopathic Medicine, Paris, France, 1994

28. Cochrane Review. Epidurals for pain relief in labour. Online document at: www.cochrane.org/CD000331/PREGepidurals-for-pain-relief-in-labour Accessed March 15, 2017.

29. Dorfman P, Lasserre MN, Tétau M. Preparation a l'accouchement par homeopathie-experimentation en double insu versus placebo. Cahier Biother 1987:94;77-81.

30. Mojaver $\mathrm{YN}$, Mosavi $\mathrm{F}$, Mazaherinezhad A, et al. Individualized homeopathic treatment of trigeminal neuralgia: An observational study. Homeopathy 2007;96:82-86.

31. Morrison R. Desktop Guide to Physical Pathology. Nevada City, CA: Hahnemann Clinic Publishing, 1998:3-26.

32. Dean ME. The Trials of Homeopathy: Origins, Structure and Development. Essen, Germany: KVC Verlag, 2004.

33. Dorsey ER, Roulet JD, Thompson JP, et al. Financial anatomy of biomedical research, 2003-2008. JAMA 2010;303:137-143.
34. Hill AB. Heberden Oration 1965: Reflections on the controlled trial. Ann Rheum Dis 1966;25:107-113.

35. Rawlins M. De testimonio: On the evidence for decisions about the use of therapeutic interventions. Lancet 2008;372:2152-2161.

36. Angell M. The Truth About Drug Companies: How They Deceive Us and What to Do About It. New York: Random House, 2005.

37. Steinsbekk A, Bentzen N, Fonnebo V, Lewith G. Self treatment with one of three self selected, ultramolecular homeopathic medicines for the prevention of upper respiratory tract infections in children. A double-blind randomized placebo controlled trial. Br J Clin Pharmacol 2005;59:447-455.

38. Schroyens F, ed. Synthesis Repertorium Homeopathicum Syntheticum. London: Homeopathic Book Publishers 1993:420.

39. Pedrero-Escalas MF, Jimenez-Antoni J, Lassaletta L, et al. Hospital clinical trial: Homeopathy (Agraphis nutans $5 \mathrm{CH}$, Thuya occidentalis $5 \mathrm{CH}$, Kalium muriaticum $9 \mathrm{CH}$ and Arsenicum iodatum $9 \mathrm{CH}$ ) as adjuvant, in children with otitis media with effusion. Int J Pediatr Otorhinolaryngol 2016; 88:217-223.

40. Stevinson C, Devaraj VS, Fountain-Barber A, et al. Homeopathic Arnica for prevention of pain and bruising: Randomized placebo-controlled trial in hand surgery. J R Soc Med 2003;96:60-65.

41. Bornhuft G, Matthiessen PF. Homeopathy in Healthcare: Effectiveness, Appropriateness, Safety, Costs. Goslar, Germany: Springer, 2011.

42. Witt CM, Ludtke R, Baur R, Willich SN. Homeopathic treatment of patients with chronic low back pain: A prospective observational study with 2 years' followup. Clin J Pain 2009;25:334-339.

43. Beer AM, Fey S, Zimmer M, et al. [Effectiveness and safety of a homeopathic drug combination in the treatment of chronic low back pain: A double-blind, randomized, placebo-controlled clinical trial.] MMW Fortschr Med 2012;154:45-57 [in German]

44. Stam C, Bonnet MS, van Haselen RA. The efficacy and safety of a homeopathic gel in the treatment of acute low back pain: A multi-centre, randomized, double-blind comparative clinical trial. Br Hom J 2001;90:21-28.

45. Morris M, Pellow J, Solomon EM, Tsele-Tebakang T. Physiotherapy and a homeopathic complex for chronic low back pain due to osteoarthritis: A randomized, controlled pilot trial. Altern Ther Health Med 2016;22:48-56.

46. Eames S, Darby P. Homeopathy and its ethical use in dentistry. Br Dent $\mathrm{J}$ 2011;210:299-301.

47. Raak C, Bussing A, Gssmann G, et al. A systematic review and metaanalysis on the use of Hypericum perforatum (St. John's wort) for pain conditions in dental practice. Homeopathy 2012;101:204-210.

48. Mazzocchi A, Montanaro F. Observational study of the use of Symphytum $5 \mathrm{CH}$ in the management of pain and swelling after dental implant surgery. Homeopathy 2012;101:211-216.

49. Hamre HJ, Mittag I, Glockmann A, et al. Pulpa dentis D30 for acute reversible pulpitis: A prospective cohort study in routine dental practice. Altern Ther 2011;17:16-21.

Burke Lennihan, RN, CCH, has been a practicing homeopath for more than 20 years and lectures on homeopathy at Massachusetts College of Pharmacy in Boston, Massachusetts, and Lesley University in Cambridge, Massachusetts.

To order reprints of this article, contact the publisher at (914) 740-2100. 